\title{
Aggregation of interacting criteria by means of the discrete Choquet integral
}

\author{
Jean-Luc Marichal \\ Department of Mathematics, 292 TMCB \\ Brigham Young University, Provo, Utah 84602, USA. \\ Email: marichal [at] math. byu.edu \\ Revised version
}

\begin{abstract}
The most often used operator to aggregate criteria in decision making problems is the classical weighted arithmetic mean. In many problems however, the criteria considered interact, and a substitute to the weighted arithmetic mean has to be adopted. Under rather natural conditions, the discrete Choquet integral is proved to be an adequate aggregation operator that extends the weighted arithmetic mean by the taking into consideration of the interaction among criteria. The axiomatic that supports the Choquet integral is presented and some subfamilies are studied.
\end{abstract}

Index Terms: multicriteria decision making, interacting criteria, Choquet integral.

\section{Introduction}

Let us consider a finite set of alternatives $A=\{a, b, c, \ldots\}$ and a finite set of criteria $N=$ $\{1, \ldots, n\}$ in a multicriteria decision making problem. Each alternative $a \in A$ is associated with a profile $x^{a}=\left(x_{1}^{a}, \ldots, x_{n}^{a}\right) \in \mathbb{R}^{n}$, where, for any $i \in N, x_{i}^{a}$ represents the partial score of $a$ related to criterion $i$. We assume that all the partial scores are defined according to the same interval scale, that is, they are defined up to the same positive linear transformation.

From the profile of any alternative $a$, one can compute a global score $M\left(x^{a}\right)$ by means of an aggregation operator $M: \mathbb{R}^{n} \rightarrow \mathbb{R}$ which takes into account the weights of the criteria. Once the global scores are computed, they can be used to rank the alternatives or select an alternative that best satisfies the given criteria. For instance the optimal alternative $a^{*} \in A$ could be selected such that

$$
M\left(x^{a^{*}}\right)=\max _{a \in A} M\left(x^{a}\right) .
$$

Until recently, the most often used aggregation operators were the weighted arithmetic means, that is, operators of the form

$$
M_{\omega}(x)=\sum_{i=1}^{n} \omega_{i} x_{i}
$$


with $\sum_{i} \omega_{i}=1$ and $\omega_{i} \geq 0$ for all $i \in N$. However, since these operators are not able to model in any understandable way an interaction among criteria, they can be used only in the presence of independent criteria. They are not appropriate for the aggregation of interacting criteria.

In order to have a flexible representation of complex interaction phenomena between criteria (e.g. positive or negative synergy between some criteria), it is useful to substitute to the weight vector $\omega$ a non-additive set function on $N$ allowing to define a weight not only on each criterion, but also on each subset of criteria. For this purpose the concept of fuzzy measure [29] has been introduced.

Now, a suitable aggregation operator, which generalizes the weighted arithmetic mean, is the discrete Choquet integral (see Definition 2.2 below), whose use in multicriteria decision making was proposed by many authors (see e.g. [7, 11] and the references therein). This integral, which is constructed from the concept of fuzzy measure, is able to take into account the interaction existing among criteria. When criteria are independent, it identifies with the weighted arithmetic mean.

The main aim of this paper is to present the Choquet integral as an appropriate extension to the weighted arithmetic mean for the aggregation of criteria. This operator offers indeed a large flexibility while keeping in some sense a linear form. Although its definition is not very intuitive, we will see that the Choquet integral can be characterized axiomatically by means of rather natural properties.

The outline of this paper is as follows. In Section 2 we present the Choquet integral and we propose an axiomatic characterization. In Section 3 we introduce the importance and interaction indices which enable us to interpret the behavior of aggregation. Finally, in Section 4 we investigate particular Choquet integrals such as the weighted arithmetic mean and the ordered weighted averaging operator.

In order to avoid a heavy notation, cardinality of subsets $S, T, \ldots$ will be denoted whenever possible by the corresponding lower case letters $s, t, \ldots$, otherwise by the standard notation $|S|,|T|, \ldots$. Moreover, we will often omit braces for singletons, e.g. writing $a(i), N \backslash i$ instead of $a(\{i\}), N \backslash\{i\}$. Also, for pairs, we will often write $i j$ instead of $\{i, j\}$, as for example $a(i j)$.

For any subset $S \subseteq N$, $e_{S}$ will denote the characteristic vector of $S$ in $\{0,1\}^{n}$, i.e., the vector of $\{0,1\}^{n}$ whose $i$ th component is 1 if and only if $i \in S$.

Finally, $\wedge$ and $\vee$ will denote the minimum and maximum operations, respectively.

\section{The discrete Choquet integral}

In this section we give the definition of the Choquet integral in the discrete case. We also present an axiomatic characterization that motivates the use of this operator in applications.

\subsection{The use of the fuzzy measures}

It is known that the fuzzy measures are able to model the dependence between criteria in many situations, whatever the nature of the dependence [18]. In fact, they were proposed by Sugeno in 1974 [29] to generalize additive measures. It seems widely accepted that additivity is not suitable as a required property of set functions in many real situations, due to the lack of additivity in many facets of human reasoning. To be able to express human subjectivity, 
Sugeno proposed to replace the additivity property by a weaker one: monotonicity, and he called these non-additive monotonic measures fuzzy measures. Let us recall the definition in the discrete case.

Definition 2.1 A fuzzy measure on $N$ is a set function $v: 2^{N} \rightarrow[0,1]$ satisfying the following conditions:

i) $v(\emptyset)=0, v(N)=1$,

ii) $S \subseteq T \Rightarrow v(S) \leq v(T)$.

The set of all fuzzy measures on $N$ will be denoted by $\mathcal{F}_{N}$ as we continue.

For any $S \subseteq N, v(S)$ can be interpreted as the weight or the degree of importance of the combination $S$ of criteria, or better, its power to make the decision alone (without the remaining criteria). Thus, in addition to the usual weights on criteria taken separately, weights on any combination of criteria are also defined. Monotonicity then means that adding a new element to a combination cannot decrease its importance. Obviously $v(N)$ has the maximal value, being one by convention.

We will assume that the weights are numerical values defined on a cardinal scale. In particular, expressions like $v(S)+v(T)$ or $v(T \cup i)-v(T)$ can be interpreted.

A fuzzy measure $v \in \mathcal{F}_{N}$ is said to be additive if $v(S \cup T)=v(S)+v(T)$ whenever $S \cap T=\emptyset$. In that case it suffices to define the $n$ coefficients (weights) $v(1), \ldots, v(n)$ to define the measure entirely.

\subsection{Definition and alternative representation}

The concept of Choquet integral was first introduced in capacity theory [3]. Its use as a (fuzzy) integral with respect to a fuzzy measure was then proposed by Höhle [13] and rediscovered later by Murofushi and Sugeno [21, 22], see also [4]. Since this integral is viewed here as an $n$-place aggregation operator, we will adopt a connective-like notation instead of the usual integral form, and the integrand will be a set of $n$ values $x_{1}, \ldots, x_{n}$ of $\mathbb{R}$.

Definition 2.2 Let $v \in \mathcal{F}_{N}$. The Choquet integral of $x: N \rightarrow \mathbb{R}$ with respect to $v$ is defined by

$$
\mathcal{C}_{v}(x):=\sum_{i=1}^{n} x_{(i)}\left[v\left(A_{(i)}\right)-v\left(A_{(i+1)}\right)\right],
$$

where $(\cdot)$ indicates a permutation on $N$ such that $x_{(1)} \leq \ldots \leq x_{(n)}$. Also $A_{(i)}=\{(i), \ldots,(n)\}$, and $A_{(n+1)}=\emptyset$.

For instance, if $x_{3} \leq x_{1} \leq x_{2}$, we have

$$
\begin{aligned}
\mathcal{C}_{v}\left(x_{1}, x_{2}, x_{3}\right)= & x_{3}[v(3,1,2)-v(1,2)] \\
& +x_{1}[v(1,2)-v(2)] \\
& +x_{2} v(2) .
\end{aligned}
$$

Thus the discrete Choquet integral is a linear expression up to a reordering of the elements. It is closely related to the discrete Lebesgue integral (weighted arithmetic mean), since both coincide when the measure is additive:

$$
\mathcal{C}_{v}(x)=\sum_{i=1}^{n} v(i) x_{i}, \quad x \in \mathbb{R}^{n} .
$$


In this sense, the Choquet integral is a generalization of the Lebesgue integral.

In the Appendix, we establish a connection between the Choquet integral and the Lovász extension of a pseudo-Boolean function, a concept used in combinatorial optimization [28]. Such a connection enables us to have a geometrical interpretation of the graph of the Choquet integral.

Now, the Möbius transform of a given fuzzy measure $v \in \mathcal{F}_{N}$ is a set function $a: 2^{N} \rightarrow \mathbb{R}$ defined by

$$
a(S)=\sum_{T \subseteq S}(-1)^{s-t} v(T), \quad S \subseteq N
$$

where $s=|S|$ and $t=|T|$. The transformation is invertible and we have (see e.g. [25])

$$
v(S)=\sum_{T \subseteq S} a(T), \quad S \subseteq N
$$

The Möbius transform often makes it possible to express a function of $v$ in a simpler form. For example, the Choquet integral is written as (see Appendix)

$$
\mathcal{C}_{v}(x)=\sum_{T \subseteq N} a(T) \bigwedge_{i \in T} x_{i}, \quad x \in \mathbb{R}^{n}
$$

Using Eq. (2), we then have

$$
\mathcal{C}_{v}(x)=\sum_{T \subseteq N} v(T) f_{T}(x), \quad x \in \mathbb{R}^{n}
$$

where

$$
f_{T}(x)=\sum_{S \supseteq T}(-1)^{s-t} \bigwedge_{i \in S} x_{i}, \quad T \subseteq N
$$

\subsection{Axiomatic characterization}

Although the Choquet integrals have become popular in the field of fuzzy sets and multicriteria decision making, there exist few axiomatic characterizations of this family in the literature. The most representative one is given by Schmeidler [26], using the concept of comonotonic additivity. However, such a characterization is not very attractive in the context of multicriteria decision making.

In [18] the author proposed an axiomatic characterization of the class of all the Choquet integrals with $n$ arguments. The statement is the following.

Theorem 2.1 The operators $M_{v}: \mathbb{R}^{n} \rightarrow \mathbb{R}\left(v \in \mathcal{F}_{N}\right)$ are

- linear w.r.t. the fuzzy measures, that is, there exist $2^{n}$ functions $f_{T}: \mathbb{R}^{n} \rightarrow \mathbb{R}(T \subseteq N)$, such that

$$
M_{v}=\sum_{T \subseteq N} v(T) f_{T}, \quad v \in F_{N}
$$

- non decreasing in each argument, 
- stable for the admissible positive linear transformations, that is,

$$
M_{v}\left(r x_{1}+s, \ldots, r x_{n}+s\right)=r M_{v}\left(x_{1}, \ldots, x_{n}\right)+s
$$

for all $x \in \mathbb{R}^{n}, r>0, s \in \mathbb{R}$.

- properly weighted by $v$, that is,

$$
M_{v}\left(e_{S}\right)=v(S), \quad S \subseteq N, v \in F_{N},
$$

if and only if $M_{v}=\mathcal{C}_{v}$ for all $v \in \mathcal{F}_{N}$.

The axioms presented in the previous characterization are natural enough in the context of multicriteria decision making. The first one is proposed to keep the aggregation model as simple as possible. The second axiom says that increasing a partial score along any criterion cannot decrease the global score. The third axiom only demands that the aggregated value is stable with respect to any change of scale. Finally, assuming that the partial score scale is embedded in $[0,1]$, the fourth axiom suggests that the weight of importance of any subset $S$ of criteria is defined as the global evaluation of the alternative that completely satisfies criteria $S$ and totally fails to satisfy the others.

The fourth axiom is fundamental. It gives an appropriate definition of the weights of subsets of criteria, interpreting them as global evaluation of particular profiles.

\section{Behavioral analysis of aggregation}

Now that we have a tool for a suitable aggregation, an important question arises: How can we interpret the behavior of the Choquet integral or that of its associated fuzzy measure ? Of course the meaning of the values $v(T)$ is not always very clear for the decision maker. These values do not give immediately the global importance of the criteria, nor the degree of interaction among them.

In fact, from a given fuzzy measure, it is possible to derive some indices or parameters that will enable us to interprete the behavior of the fuzzy measure. These indices constitute a kind of identity card of the fuzzy measure. In this section, we present two types of indices: importance and interaction. Other indices, such as tolerance and dispersion, were proposed and studied by the author in $[15,16]$.

\subsection{Importance indices}

The overall importance of a criterion $i \in N$ into a decision problem is not solely determined by the number $v(i)$, but also by all $v(T)$ such that $i \in T$. Indeed, we may have $v(i)=0$, suggesting that element $i$ is unimportant, but it may happen that for many subsets $T \subseteq N, v(T \cup i)$ is much greater than $v(T)$, suggesting that $i$ is actually an important element in the decision.

Shapley [27] proposed in 1953 a definition of a coefficient of importance, based on a set of reasonable axioms. The importance index or Shapley value of criterion $i$ with respect to $v$ is defined by

$$
\phi(v, i):=\sum_{T \subseteq N \backslash i} \frac{(n-t-1) ! t !}{n !}[v(T \cup i)-v(T)]
$$


The Shapley value is a fundamental concept in game theory expressing a power index. It can be interpreted as a weighted average value of the marginal contribution $v(T \cup i)-v(T)$ of element $i$ alone in all combinations. To make this clearer, it is informative to rewrite the index as follows:

$$
\phi(v, i)=\frac{1}{n} \sum_{t=0}^{n-1} \frac{1}{\left(\begin{array}{c}
n-1 \\
t
\end{array}\right)} \sum_{\substack{T \subseteq N \backslash i \\
|T|=t}}[v(T \cup i)-v(T)] .
$$

Thus, the average value of $v(T \cup i)-v(T)$ is computed first over the subsets of same size $t$ and then over all the possible sizes. Consequently, the subsets containing about $n / 2$ criteria are the less important in the average, since they are numerous and each criterion is very often involved into them.

The use of the Shapley value in multicriteria decision making was proposed in 1992 by Murofushi [19]. It is worth noting that a basic property of the Shapley value is

$$
\sum_{i=1}^{n} \phi(v, i)=1
$$

Note also that, when $v$ is additive, we clearly have $v(T \cup i)-v(T)=v(i)$ for all $i \in N$ and all $T \subseteq N \backslash i$, and hence

$$
\phi(v, i)=v(i), \quad i \in N .
$$

If $v$ is non-additive then some criteria are dependent and (5) generally does not hold anymore. This shows that it is sensible to search for a coefficient of overall importance for each criterion.

In terms of the Möbius representation, the Shapley value takes a very simple form [27]:

$$
\phi(v, i)=\sum_{T \ni i} \frac{1}{t} a(T) .
$$

Now, since the Shapley indices are non-negative and sum up to one, it would be interesting to consider the weighted arithmetic mean having these indices as weights. We call this operator the Shapley integral [18].

Definition 3.1 Let $v \in \mathcal{F}_{N}$. The Shapley integral of $x: N \rightarrow \mathbb{R}$ with respect to $v$ is defined by

$$
\operatorname{Sh}_{v}(x)=\sum_{i \in N} \phi(v, i) x_{i}
$$

This operator will play an important role in Theorem 4.1 (Section 4.3).

Now, the concept of importance index can be easily generalized to subsets of criteria. The importance index of subset $S \subseteq N$ with respect to $v$ is defined by

$$
\phi(v, S):=\sum_{T \subseteq N \backslash S} \frac{(n-t-s) ! t !}{(n-s+1) !}[v(T \cup S)-v(T)] .
$$

This index, introduced by the author in [17] as the influence index of criteria $S$, measures the overall importance of subset $S$ of criteria. 
In terms of the Möbius representation, it is given by

$$
\phi(v, S)=\sum_{\substack{T \subseteq N \\ T \cap S \neq \emptyset}} \frac{1}{|T \backslash S|+1} a(T) .
$$

It was shown [17] that this expression is also the average amplitude of the range of $\mathcal{C}_{v}$ that criteria $S$ may control when assigning partial scores in $[0,1]$ to the criteria not in $S$ at random. That is,

$$
\begin{aligned}
\phi(v, S) & =\int_{0}^{1} \cdots \int_{0}^{1}\left[\lim _{\substack{x_{j} \rightarrow 1 \\
j \in S}} \mathcal{C}_{v}(x)-\lim _{\substack{x_{j} \rightarrow 0 \\
j \in S}} \mathcal{C}_{v}(x)\right] \mathrm{d} x_{i_{1}} \cdots \mathrm{d} x_{i_{n-s}} \\
& =\int_{[0,1]^{n}}\left[\lim _{\substack{x_{j} \rightarrow 1 \\
j \in S}} \mathcal{C}_{v}(x)-\lim _{\substack{x_{j} \rightarrow 0 \\
j \in S}} \mathcal{C}_{v}(x)\right] \mathrm{d} x,
\end{aligned}
$$

where $N \backslash S=\left\{i_{1}, \ldots, i_{n-s}\right\}$.

\subsection{Interaction indices}

Another interesting concept is that of interaction among criteria. We have seen that when the fuzzy measure is not additive then some criteria interact. Of course, it would be interesting to appraise the degree of interaction among any subset of criteria.

Consider first a pair $\{i, j\} \subseteq N$ of criteria. It may happen that $v(i)$ and $v(j)$ are small and at the same time $v(i j)$ is large. Clearly, the number $\phi(v, i)$ merely measures the average contribution that criterion $i$ brings to all possible combinations, but it gives no information on the phenomena of interaction existing among criteria.

Clearly, if the marginal contribution of $j$ to every combination of criteria that contains $i$ is greater (resp. less) than the marginal contribution of $j$ to the same combination when $i$ is excluded, then the expression

$$
[v(T \cup i j)-v(T \cup i)]-[v(T \cup j)-v(T)]
$$

is positive (resp. negative) for any $T \subseteq N \backslash i j$. We then say that $i$ and $j$ positively (resp. negatively) interact.

This latter expression is called the marginal interaction between $i$ and $j$, conditioned to the presence of elements of the combination $T \subseteq N \backslash i j$. Now, an interaction index for $\{i, j\}$ is given by an average value of this marginal interaction. Murofushi and Soneda [20] proposed in 1993 to calculate this average value as for the Shapley value. Setting

$$
\left(\Delta_{i j} v\right)(T):=v(T \cup i j)-v(T \cup i)-v(T \cup j)+v(T),
$$

the interaction index of criteria $i$ and $j$ related to $v$ is then defined by

$$
I(v, i j):=\sum_{T \subseteq N \backslash i j} \frac{(n-t-2) ! t !}{(n-1) !}\left(\Delta_{i j} v\right)(T) .
$$


It should be mentioned that, historically, the interaction index (6) was first introduced in 1972 by Owen (see Eq. (28) in [24]) in game theory to express a degree of complementarity or competitiveness between elements $i$ and $j$.

The interaction index among a combination $S$ of criteria was introduced by Grabisch [8] as a natural extension of the case $s=2$. The interaction index of $S(s \geq 2)$ related to $v$, is defined by

$$
I(v, S):=\sum_{T \subseteq N \backslash S} \frac{(n-t-s) ! t !}{(n-s+1) !}\left(\Delta_{S} v\right)(T),
$$

where we have set

$$
\left(\Delta_{S} v\right)(T):=\sum_{L \subseteq S}(-1)^{s-l} v(L \cup T) .
$$

In terms of the Möbius representation, this index is written [8]

$$
I(v, S)=\sum_{T \supseteq S} \frac{1}{t-s+1} a(T), \quad S \subseteq N .
$$

Viewed as a set function, it coincides on singletons with the Shapley value (4).

In terms of the Choquet integral, we have [10, Proposition 4.1]

$$
\begin{aligned}
I(v, S) & =\int_{0}^{1} \cdots \int_{0}^{1}\left(\Delta_{S} \mathcal{C}_{v}\right)(x) \mathrm{d} x_{i_{1}} \cdots \mathrm{d} x_{i_{n-s}} \\
& =\int_{[0,1]^{n}}\left(\Delta_{S} \mathcal{C}_{v}\right)(x) \mathrm{d} x,
\end{aligned}
$$

where $N \backslash S=\left\{i_{1}, \ldots, i_{n-s}\right\}$ and

$$
\left(\Delta_{S} \mathcal{C}_{v}\right)(x):=\sum_{L \subseteq S}(-1)^{s-l} \lim _{\substack{x_{i} \rightarrow 1 \\ i \in L}} \lim _{\substack{x_{j} \rightarrow 0 \\ j \in S \backslash L}} \mathcal{C}_{v}(x) .
$$

It was also proved [10, Proposition 5.1] that the transformation (7) is invertible and its inverse is written as

$$
a(S)=\sum_{T \supseteq S} B_{t-s} I(v, T), \quad S \subseteq N
$$

where $B_{n}$ is the $n$th Bernoulli number, that is the $n$th element of the numerical sequence $\left\{B_{n}\right\}_{n \in \mathbb{N}}$ defined recursively by

$$
\left\{\begin{array}{l}
B_{0}=1, \\
\sum_{k=0}^{n}\left(\begin{array}{c}
n+1 \\
k
\end{array}\right) B_{k}=0, \quad n \in \mathbb{N} \backslash\{0\} .
\end{array}\right.
$$

\section{Particular Choquet integrals}

We have seen that the Choquet integral with respect to an additive measure is a weighted arithmetic mean. Of course, they are also other interesting particular Choquet integrals, such as symmetric Choquet integrals. In this section we investigate some of them.

Any vector $\omega \in[0,1]^{n}$ such that $\sum_{i} \omega_{i}=1$ will be called a weight vector as we continue. 


\subsection{The weighted arithmetic mean}

Definition 4.1 For any weight vector $\omega \in[0,1]^{n}$, the weighted arithmetic mean operator WAM $_{\omega}$ associated to $\omega$ is defined by

$$
\operatorname{WAM}_{\omega}(x)=\sum_{i=1}^{n} \omega_{i} x_{i} .
$$

We have seen that $\mathrm{WAM}_{\omega}$ is a Choquet integral $\mathcal{C}_{v}$ with respect to an additive fuzzy measure:

$$
v(S)=\sum_{i \in S} \omega_{i}, \quad S \subseteq N
$$

The corresponding Möbius representation is given by

$$
\left\{\begin{array}{l}
a(i)=\omega_{i}, \quad \forall i \in N \\
a(S)=0, \quad \forall S \subseteq N \text { such that } s \geq 2 .
\end{array}\right.
$$

Conversely, the weights associated to $\mathrm{WAM}_{\omega}$ are given by

$$
\omega_{i}=v(i)=a(i), \quad i \in N .
$$

Furthermore the importance and interaction indices of $v$ are written

$$
\begin{array}{ll}
\phi(v, i)=\omega_{i} \quad \forall i \in N, \\
\phi(v, S)=\sum_{i \in S} \omega_{i} \quad \forall S \subseteq N \\
I(v, S)=0 \quad \forall S \subseteq N \text { such that } s \geq 2 .
\end{array}
$$

The class of weighted arithmetic mean $\mathrm{WAM}_{\omega}$ includes two important special cases, namely:

- the arithmetic mean

$$
\operatorname{AM}(x)=\frac{1}{n} \sum_{i=1}^{n} x_{i}
$$

when $\omega_{i}=1 / n$ for all $i \in N$. In this case, we have $v(S)=\phi(v, S)=s / n$ for all $S \subseteq N$ and $a(i)=\phi(v, i)=1 / n$ for all $i \in N$.

- the $k$ th projection

$$
\mathrm{P}_{k}(x)=x_{k},
$$

when $\omega_{k}=1$ for some $k \in N$. In this case, we have $v(S)=\phi(v, S)=1$ if $S \ni k$ and 0 otherwise. Moreover, we have $a(i)=\phi(v, i)=1$ if $i=k$ and 0 otherwise. 


\subsection{The ordered weighted averaging}

Yager [30] defined in 1988 the ordered weighted averaging operators (OWA) as follows.

Definition 4.2 For any weight vector $\omega \in[0,1]^{n}$, the ordered weighted averaging operator $\mathrm{OWA}_{\omega}$ associated to $\omega$ is defined by

$$
\operatorname{OWA}_{\omega}(x)=\sum_{i=1}^{n} \omega_{i} x_{(i)}
$$

with the convention that $x_{(1)} \leq \cdots \leq x_{(n)}$.

It was proved in $[23, \S 4]$ and [6] that the OWA operators coincides with the subclass of discrete Choquet integrals that are symmetric. We give below a rather short proof of this result.

Proposition 4.1 Let $v \in \mathcal{F}_{N}$. Then the following assertions are equivalent.

i) $v(S)=v\left(S^{\prime}\right)$ whenever $|S|=\left|S^{\prime}\right|$.

ii) $\exists \omega \in[0,1]^{n}$ such that $\mathcal{C}_{v}=\mathrm{OWA}_{\omega}$.

iii) $\mathcal{C}_{v}$ is a symmetric function.

Proof. $i) \Rightarrow i i$ ) Since $v$ depends only on the cardinality of subsets, the difference

$$
v\left(A_{(i)}\right)-v\left(A_{(i+1)}\right)
$$

depends only on $i$. Denoting this difference by $\omega_{i}$, we have by (1),

$$
\mathcal{C}_{v}(x)=\sum_{i=1}^{n} \omega_{i} x_{(i)}, \quad x \in \mathbb{R}^{n}
$$

with $\sum_{i} \omega_{i}=1$ and $\omega_{i} \geq 0$ for all $i \in N$.

ii) $\Rightarrow$ iii) Trivial.

iii) $\Rightarrow$ i) Fix $S, S^{\prime} \subseteq N$ such that $|S|=\left|S^{\prime}\right|$. Of course, there exists a permutation $\pi$ of $N$ such that $S^{\prime}=\pi(S)$, where $\pi(S):=\{\pi(i) \mid i \in S\}$. Now, by symmetry, we have

$$
v(S)=\mathcal{C}_{v}\left(e_{S}\right)=\mathcal{C}_{v}\left(e_{\pi(S)}\right)=v(\pi(S))=v\left(S^{\prime}\right)
$$

which completes the proof.

The fuzzy measure $v$ associated to $\mathrm{OWA}_{\omega}$ is given by

$$
v(S)=\sum_{i=n-s+1}^{n} \omega_{i}, \quad S \subseteq N, \quad S \neq \emptyset,
$$

and its Möbius representation by [9, Theorem 1]

$$
a(S)=\sum_{j=0}^{s-1}\left(\begin{array}{c}
s-1 \\
j
\end{array}\right)(-1)^{s-1-j} \omega_{n-j}, \quad S \subseteq N, \quad S \neq \emptyset .
$$


Conversely, the weights associated to $\mathrm{OWA}_{\omega}$ are given by

$$
\omega_{n-s}=v(S \cup i)-v(S)=\sum_{T \subseteq S} a(T \cup i), \quad i \in N, S \subseteq N \backslash i .
$$

Now, let us show that the Möbius representation can take a very simple form in terms of $\omega$. Consider the difference operator

$$
\Delta_{k} x_{k}:=x_{k+1}-x_{k}
$$

for sequences $\left(x_{k}\right)_{k \in \mathbb{N}}$. Denoting by $\Delta_{k}^{s}$ the $s$ th iterate of $\Delta_{k}$, it is well-known that we have (cf. Berge [1, Chap. 1, Sect. 8])

$$
\Delta_{k}^{s} x_{k}=\sum_{j=0}^{s}\left(\begin{array}{l}
s \\
j
\end{array}\right)(-1)^{s-j} x_{k+j}, \quad s \in \mathbb{N} .
$$

We then have

$$
a(S)=\left[\Delta_{k}^{s-1} \omega_{n-k}\right]_{k=0}, \quad S \subseteq N, \quad S \neq \emptyset .
$$

According Proposition 4.1, the use of a symmetric Choquet integral means that all the criteria have the same importance. More precisely, all the combinations of criteria of the same size have the same importance. Notice that this not imply that criteria do not interact. The following result, proved in [9, Theorem 1], shows that symmetric criteria can be dependent. We give below a more concise and precise proof of it.

Proposition 4.2 Let $\omega \in[0,1]^{n}$ be a weight vector and let $v \in \mathcal{F}_{N}$ such that $\mathcal{C}_{v}=\mathrm{OWA}_{\omega}$. Then

$$
\phi(v, i)=1 / n, \quad i \in N,
$$

and, for all $S \subseteq N$ such that $s \geq 2$,

$$
I(v, S)=\frac{1}{n-s+1} \sum_{j=0}^{s-2}\left(\begin{array}{c}
s-2 \\
j
\end{array}\right)(-1)^{s-j}\left(\omega_{s-j-1}-\omega_{n-j}\right) .
$$

Proof. By Proposition 4.1, we can set $v_{t}:=v(T)$ for all $T \subseteq N$. Next, for any $S \subseteq N$, $S \neq \emptyset$, and any $T \subseteq N \backslash S$, we have, by (9) and (10),

$$
\begin{aligned}
\sum_{L \subseteq S}(-1)^{s-l} v(L \cup T) & =\sum_{l=0}^{s}\left(\begin{array}{l}
s \\
l
\end{array}\right)(-1)^{s-l} v_{l+t} \\
& =\Delta_{t}^{s} v_{t} \\
& =\Delta_{t}^{s-1}\left(v_{t+1}-v_{t}\right) \\
& =\Delta_{t}^{s-1} \omega_{n-t},
\end{aligned}
$$

and hence, by definition of $I(v, S)$,

$$
\begin{aligned}
I(v, S) & =\sum_{T \subseteq N \backslash S} \frac{(n-t-s) ! t !}{(n-s+1) !} \Delta_{t}^{s-1} \omega_{n-t} \\
& =\frac{1}{n-s+1} \sum_{t=0}^{n-s} \Delta_{t}^{s-1} \omega_{n-t} .
\end{aligned}
$$


If $S=\{i\}$ for some $i \in N$, we have

$$
\phi(v, i)=I(v, i)=\frac{1}{n} \sum_{t=0}^{n-1} \omega_{n-t}=\frac{1}{n} .
$$

If $s \geq 2$, we can set $\lambda_{t}:=\Delta_{t}^{s-2} \omega_{n-t}$ and we have

$$
\begin{aligned}
I(v, S) & =\frac{1}{n-s+1} \sum_{t=0}^{n-s} \Delta_{t} \lambda_{t} \\
& =\frac{1}{n-s+1} \sum_{t=0}^{n-s}\left(\lambda_{t+1}-\lambda_{t}\right) \\
& =\frac{1}{n-s+1}\left(\lambda_{n-s+1}-\lambda_{0}\right) .
\end{aligned}
$$

Another application of (10) then leads to the result.

The previous result shows that $I(v, S)$ depends only on $s$, so that the interaction among criteria is constant on all subsets of the same size. For example, for any pair $\{i, j\} \subseteq N$, we have

$$
I(v, i j)=\frac{\omega_{1}-\omega_{n}}{n-1} .
$$

Regarding the importance of subsets of criteria, we have the following formula [17, Proposition 6.2]:

$$
\phi(v, S)=\sum_{i=1}^{n} \omega_{i} \frac{i \wedge s \wedge(n-i+1) \wedge(n-s+1)}{n-s+1}, \quad S \subseteq N .
$$

The class of ordered weighted averaging operators $\mathrm{OWA}_{\omega}$ includes some important special cases, namely:

- the arithmetic mean

$$
\operatorname{AM}(x)=\frac{1}{n} \sum_{i=1}^{n} x_{i}
$$

when $\omega_{i}=1 / n$ for all $i \in N$.

- the $k$ th order statistic

$$
\mathrm{OS}_{k}(x)=x_{(k)},
$$

when $\omega_{k}=1$ for some $k \in N$. In this case, we have

$$
\begin{aligned}
& v(S)= \begin{cases}1 & \text { if } s \geq n-k+1 \\
0 & \text { otherwise }\end{cases} \\
& a(S)= \begin{cases}(-1)^{s-n+k-1}\left(\begin{array}{l}
s-1 \\
n-k
\end{array}\right) & \text { if } s \geq n-k+1 \\
0 & \text { otherwise }\end{cases}
\end{aligned}
$$

Moreover, we have

$$
\begin{array}{ll}
\phi(v, S)=\frac{k \wedge s \wedge(n-k+1) \wedge(n-s+1)}{n-s+1} & \forall S \subseteq N, \\
I(v, S)=\frac{(-1)^{k+1}}{n-s+1}\left[\left(\begin{array}{c}
s-2 \\
n-k
\end{array}\right)(-1)^{n-s}+\left(\begin{array}{c}
s-2 \\
s-k-1
\end{array}\right)\right] & \forall S \subseteq N \text { such that } s \geq 2,
\end{array}
$$


with the convention that $\left(\begin{array}{l}p \\ q\end{array}\right)=0$ whenever $p<q$ or $q<0$.

In particular, $I(v, i j)=0$ whenever $k \neq 1$ and $k \neq n$. Thus there exist non-additive fuzzy measures that present no interaction between pairs of criteria.

- the min operator

$$
\min (x)=\min _{i \in N} x_{i}
$$

when $\omega_{1}=1$. In this case, we have $v(S)=a(S)=1$ if $S=N$ and 0 otherwise. Moreover,

$$
\phi(v, S)=I(v, S)=\frac{1}{n-s+1}, \quad S \subseteq N, S \neq \emptyset .
$$

- the max operator

$$
\max (x)=\max _{i \in N} x_{i}
$$

when $\omega_{n}=1$. In this case, we have $v(S)=1$ and $a(S)=(-1)^{s-1}$ for all $S \neq \emptyset$. Moreover,

$$
\phi(v, S)=\frac{1}{n-s+1} \text { and } I(v, S)=\frac{(-1)^{s-1}}{n-s+1}, \quad S \subseteq N, S \neq \emptyset .
$$

\subsection{The 2-order Choquet integrals}

We know that a problem involving $n$ criteria requires $2^{n}$ coefficients in $[0,1]$ in order to define the fuzzy measure $v$ on every subset. Of course, a decision maker is not able to give such an amount of information. To overcome this problem, Grabisch [8] proposed to approximate $v$ by a $k$-order fuzzy measure.

Definition $4.3 v \in \mathcal{F}_{N}$ is said to be of order $k(k \leq n)$ if its corresponding Möbius representation a fulfils $a(T)=0$ for all $T$ such that $t>k$, and there exists at least one subset $K$ of $k$ elements such that $a(K) \neq 0$.

This concept allows us to range freely between purely additive measures $(k=1)$, defined by $n$ coefficients, and general fuzzy measures $(k=n)$, defined by $2^{n}$ coefficients. Indeed, when varying $k$ from 1 to $n$, we recover all possible fuzzy measures. Looking at Eq. (7)-(8), we see that considering $k$-order fuzzy measures merely amounts to assuming that the interactions $I(v, T)$ for $t>k$ are zero.

Although the additive model is very simple to handle (only $n$ coefficients are needed), it is restrictive and leads to a very poor modeling tool for application in multicriteria decision making. Grabisch [8] then suggested to consider the 2-order case, which seems to be the most interesting in practical applications, since it permits to model somehow interaction among criteria while remaining very simple. Indeed, only $n+\left(\begin{array}{l}n \\ 2\end{array}\right)=\frac{n(n+1)}{2}$ coefficients are required to define the fuzzy measure, namely the coefficients

$$
\begin{aligned}
v(i) & =a(i), \quad i \in N, \\
v(i j) & =a(i)+a(j)+a(i j), \quad\{i, j\} \subseteq N .
\end{aligned}
$$


The other coefficients are then given by

$$
\begin{aligned}
v(S) & =\sum_{i \in S} a(i)+\sum_{\{i, j\} \subseteq S} a(i j) \\
& =\sum_{\{i, j\} \subseteq S} v(i j)-(s-2) \sum_{i \in S} v(i),
\end{aligned}
$$

for all $S \subseteq N$ such that $s \geq 2$. Moreover, we have immediately

$$
\begin{aligned}
\phi(v, i) & =a(i)+\frac{1}{2} \sum_{j \in N \backslash i} a(i j), \quad i \in N, \\
\phi(v, S) & =\sum_{i \in S} \phi(v, i), \quad S \subseteq N, \\
I(v, i j) & =a(i j), \quad i, j \in N,
\end{aligned}
$$

and $I(v, S)=0$ for all $S \subseteq N$ such that $s>2$.

The Choquet integral with respect to a 2-order fuzzy measure is then written as

$$
C_{v}(x)=\sum_{i \in N} a(i) x_{i}+\sum_{\{i, j\} \subseteq N} a(i j)\left(x_{i} \wedge x_{j}\right) .
$$

The following result presents this Choquet integral in terms of the interaction indices. This will allow us to interpret the aggregation process more easily.

Theorem 4.1 Let $v \in \mathcal{F}_{N}$ of order 2. Then the best weighted arithmetic mean $\mathrm{WAM}_{\omega}$ that minimizes

$$
\int_{[0,1]^{n}}\left[\mathcal{C}_{v}(x)-\operatorname{WAM}_{\omega}(x)\right]^{2} d x
$$

is given by the Shapley integral $\mathrm{Sh}_{v}$. Moreover, we have

$$
\mathcal{C}_{v}(x)=\operatorname{Sh}_{v}(x)-\frac{1}{2} \sum_{\{i, j\} \subseteq N} I(v, i j)\left|x_{i}-x_{j}\right|, \quad x \in \mathbb{R}^{n} .
$$

Proof. The first part has been proved in [15, Sect. 7.3].

Now, by using Eq. (8), we have, for all $x \in \mathbb{R}^{n}$,

$$
\begin{aligned}
\mathcal{C}_{v}(x) & =\sum_{\substack{T \subseteq N \\
t \geq 1}} a(T) \bigwedge_{i \in T} x_{i} \\
& =\sum_{\substack{T \subseteq N \\
t \geq 1}}\left[\sum_{\substack{K \supseteq T \\
k \geq 1}} B_{k-t} I(v, K)\right] \bigwedge_{i \in T} x_{i} \\
& =\sum_{\substack{K \subseteq N \\
k \geq 1}}\left[\sum_{\substack{T \subseteq K \\
t \geq 1}} B_{k-t} \bigwedge_{i \in T} x_{i}\right] I(v, K) .
\end{aligned}
$$

Since $I(v, K)=0$ whenever $k \geq 3$, we simply have

$$
\begin{aligned}
& \mathcal{C}_{v}(x) \\
& =\operatorname{Sh}_{v}(x)+\sum_{\{i, j\} \subseteq N}\left[-\frac{1}{2} x_{i}-\frac{1}{2} x_{j}+x_{i} \wedge x_{j}\right] I(v, i j) \\
& =\operatorname{Sh}_{v}(x)-\frac{1}{2} \sum_{\{i, j\} \subseteq N} I(v, i j)\left[\left(x_{i} \vee x_{j}\right)-\left(x_{i} \wedge x_{j}\right)\right],
\end{aligned}
$$


as expected.

Equation (17) shows a decomposition of the Choquet integral $\mathcal{C}_{v}$ into a linear part and a non-linear part. The linear part, namely the Shapley integral, appears to be a first order approximation of $\mathcal{C}_{v}$. The non-linear part brings some correction to the Shapley integral that we can interpret in terms of interaction as follows. Consider a pair of criteria $\{i, j\} \subseteq N$.

- A negative $I(v, i j)$, that is $I(v, i j) \in[-1,0]$, implies a disjunctive behavior between $i$ and $j$. If only one criterion of the two is satisfied then the linear average should be improved by a positive amount that is proportional to the gap between the scores $x_{i}$ and $x_{j}$ but also to the amplitude of the interaction $|I(v, i j)|$.

- A positive $I(v, i j)$, that is $I(v, i j) \in[0,1]$, implies a conjunctive behavior. In case of unilateral satisfaction, the linear average should be decreased by the same quantity as explained in the first case.

Now, regarding OWA operators, we also have the following.

Proposition 4.3 The operator $\mathrm{OWA}_{\omega}$ is a Choquet integral defined from a 2-order fuzzy measure if and only if $\omega_{s}$ is linear in $s$, that is, $\omega_{s}=\alpha s+\beta$ for some $\alpha, \beta \in \mathbb{R}$.

Proof. (Necessity) By (9), we simply have $\omega_{s}=a(i)+(n-s) a(i j)$ for all $i, j, s \in N$.

(Sufficiency) By (11), we have

$$
a(S)=\left[\Delta_{k}^{s-1} \alpha(n-k)+\beta\right]_{k=0}, \quad S \subseteq N, S \neq \emptyset,
$$

and hence $a(S)=0$ whenever $s>2$.

Theorem 4.2 Any $\mathrm{OWA}_{\omega}$ of order 2 is of the form

$$
\operatorname{OWA}_{\omega}(x)=\frac{1}{n} \sum_{i=1}^{n} x_{i}+\frac{1}{2} \frac{\omega_{1}-\omega_{n}}{n-1} \sum_{i=1}^{n}(n+1-2 i) x_{(i)},
$$

for all $x \in \mathbb{R}^{n}$.

Proof. By Eq. (12)-(16), we immediately have

$$
\begin{aligned}
a(i) & =\frac{1}{n}-\frac{1}{2}\left(\omega_{1}-\omega_{n}\right), \quad i \in N, \\
a(i j) & =\frac{\omega_{1}-\omega_{n}}{n-1}, \quad i, j \in N,
\end{aligned}
$$

and by (9), we have

$$
\begin{aligned}
\omega_{s} & =a(i)+(n-s) a(i j) \\
& =\frac{1}{n}+\frac{\omega_{1}-\omega_{n}}{2(n-1)}(n+1-2 s),
\end{aligned}
$$

for all $i, j, s \in N$, which is sufficient.

Interestingly enough, comparing Eq. (17) and (18) leads to the following remarkable identity:

$$
\sum_{\{i, j\} \subseteq N}\left|x_{i}-x_{j}\right|=\sum_{i=1}^{n}(2 i-n-1) x_{(i)}, \quad x \in \mathbb{R}^{n} .
$$




\section{Concluding remarks}

The Choquet integral is an appropriate substitute to the weighted arithmetic mean to aggregate dependent decision criteria. The motivation is based mainly on an axiomatic characterization of the class of Choquet integrals, but also on an intuitive interpretation of its expression, especially in the 2-order case.

We hope that this will encourage people of the multicriteria decision making community to use this innovative technique of aggregation, which seems very promising.

\section{Appendix: Pseudo-Boolean functions and their Lovász extensions}

Any set function $v: 2^{N} \rightarrow \mathbb{R}$ can be assimilated unambiguously with a pseudo-Boolean function $f:\{0,1\}^{n} \rightarrow \mathbb{R}$. The correspondence is straightforward: We have

$$
f(x)=\sum_{T \subseteq N} v(T) \prod_{i \in T} x_{i} \prod_{i \notin T}\left(1-x_{i}\right), \quad x \in\{0,1\}^{n},
$$

and $v(S)=f\left(e_{S}\right)$ for all $S \subseteq N$.

In particular, any pseudo-Boolean function that corresponds to a fuzzy measure is increasing in each variable and fulfils the boundary conditions: $f\left(e_{\emptyset}\right)=0$ and $f\left(e_{N}\right)=1$.

Hammer and Rudeanu [12] showed that any pseudo-Boolean function has a unique expression as a multilinear polynomial in $n$ variables:

$$
f(x)=\sum_{T \subseteq N} a(T) \prod_{i \in T} x_{i}, \quad x \in\{0,1\}^{n},
$$

with $a(T) \in \mathbb{R}$ for all $T \subseteq N$. These coefficients $a(T)$ correspond to the Möbius transform of $v$, see (3). Indeed, we have

$$
v(S)=f\left(e_{S}\right)=\sum_{T \subseteq S} a(T)
$$

for every $S \subseteq N$.

Lovász $\left[14\right.$, Sect. 3] observed that any $x \in\left(\mathbb{R}^{+}\right)^{n} \backslash\left\{e_{\emptyset}\right\}$ can be written uniquely in the form

$$
x=\sum_{i=1}^{k} \lambda_{i} e_{S_{i}}
$$

where $\lambda_{1}, \ldots, \lambda_{k}>0$ and $\emptyset \neq S_{1} \varsubsetneqq \cdots \varsubsetneqq S_{k} \subseteq N$. For example, we have

$$
\begin{aligned}
& (1,5,3)=2(0,1,0)+2(0,1,1)+1(1,1,1) \\
& (0,5,3)=2(0,1,0)+3(0,1,1) .
\end{aligned}
$$

Hence any function $f:\{0,1\}^{n} \rightarrow \mathbb{R}$ with $f\left(e_{\emptyset}\right)=0$ can be extended to $\hat{f}:\left(\mathbb{R}^{+}\right)^{n} \rightarrow \mathbb{R}$, by $\hat{f}\left(e_{\emptyset}\right)=0$ and

$$
\hat{f}(x)=\sum_{i=1}^{k} \lambda_{i} f\left(e_{S_{i}}\right) \quad\left(x=\sum_{i=1}^{k} \lambda_{i} e_{S_{i}} \in\left(\mathbb{R}^{+}\right)^{n} \backslash\left\{e_{\emptyset}\right\}\right) .
$$


Indeed, $\hat{f}$ is well defined (due to the uniqueness of (19)) and $\hat{f}(x)=f(x)$ for all $x \in\{0,1\}^{n}$. The function $\hat{f}$ is called [5] the Lovász extension of $f$.

Now, the Lovász extension of an arbitrary function $f:\{0,1\}^{n} \rightarrow \mathbb{R}$ is defined by

$$
\hat{f}(x)=f\left(e_{\emptyset}\right)+\hat{f}_{0}(x), \quad x \in\left(\mathbb{R}^{+}\right)^{n},
$$

where $\hat{f}_{0}$ is the Lovász extension of $f_{0}=f-f\left(e_{\emptyset}\right)$.

The hypercube $[0,1]^{n}$ can be subdivided into $n$ ! simplices $\mathcal{B}_{\pi}$ of the form

$$
\mathcal{B}_{\pi}:=\left\{x \in[0,1]^{n} \mid x_{\pi(1)} \leq \cdots \leq x_{\pi(n)}\right\}, \quad \pi \in \Pi,
$$

where $\Pi$ is the set of all permutations of $N$.

It is well known [28] that $\mathcal{B}_{\pi}$ is a polytope with vertices $\varepsilon_{i}^{\pi}=e_{\{\pi(i), \ldots, \pi(n)\}}(i=1, \ldots, n+1)$. Singer [28, Sect. 2] showed that $\hat{f}$ is defined on each cone $\mathcal{K}_{\pi}=\left\{\lambda \mathcal{B}_{\pi} \mid \lambda \geq 0\right\}$ as the unique affine function that coincides with $f$ at the $n+1$ vertices of $\mathcal{B}_{\pi}$. More formally, $f$ can be written as

$$
\hat{f}(x)=f\left(e_{\emptyset}\right)+\sum_{i=1}^{n} x_{\pi(i)}\left[f\left(\varepsilon_{i}^{\pi}\right)-f\left(\varepsilon_{i+1}^{\pi}\right)\right], \quad x \in \mathcal{K}_{\pi}
$$

Geometrically, on each simplex $\mathcal{B}_{\pi}$, the graph of $\hat{f}$ is the portion of the unique hyperplane passing through $\left(\varepsilon_{1}^{\pi}, f\left(\varepsilon_{1}^{\pi}\right)\right), \ldots,\left(\varepsilon_{n+1}^{\pi}, f\left(\varepsilon_{n+1}^{\pi}\right)\right)$ (which is, actually, their convex hull). According to Singer [28], the restriction of $\hat{f}$ to $[0,1]^{n}$ is called the tight extension of $f$ associated to the standard triangulation $\left\{\mathcal{B}_{\pi} \mid \pi \in \Pi\right\}$ of $[0,1]^{n}$.

A practical form of $\hat{f}$ is given by the following result.

Proposition 6.1 The Lovász extension of $f:\{0,1\}^{n} \rightarrow \mathbb{R}$ is given by

$$
\hat{f}(x)=\sum_{T \subseteq N} a(T) \bigwedge_{i \in T} x_{i}, \quad x \in\left(\mathbb{R}^{+}\right)^{n},
$$

where the coefficients $a(T)$ are those of the unique multilinear expression of $f$.

Proof. The function (21) agrees with $f$ at all the vertices of $[0,1]^{n}$, and identifies with an affine function on each cone $\mathcal{K}_{\pi}$, which is sufficient.

Let $v \in \mathcal{F}_{N}$. By $(20)$, we immediately see that the Choquet integral $\mathcal{C}_{v}$, defined on $\left(\mathbb{R}^{+}\right)^{n}$, is nothing else than the Lovász extension of the pseudo-Boolean function $f$ which represents $v$ :

$$
\mathcal{C}_{v}=\hat{f} \quad \text { on }\left(\mathbb{R}^{+}\right)^{n} .
$$

Moreover, since the expression in (21) is stable on $\mathbb{R}^{n}$ for the positive linear transformations (cf. Theorem 2.1), it corresponds to the Choquet integral. Indeed, any function from $\mathbb{R}^{n}$ to $\mathbb{R}$ fulfilling this property is completely defined by its restriction on $[0,1]^{n}$, see Proposition 3.1 in [18]. We then have the following result (see also [2]).

Proposition 6.2 Any Choquet integral $\mathcal{C}_{v}: \mathbb{R}^{n} \rightarrow \mathbb{R}$ can be written as

$$
\mathcal{C}_{v}(x)=\sum_{T \subseteq N} a(T) \bigwedge_{i \in T} x_{i}, \quad x \in \mathbb{R}^{n}
$$

where $a$ is the Möbius representation of $v$. 
Thus, the Choquet integral $\mathcal{C}_{v}$ is a piecewise affine function on $\mathbb{R}^{n}$, which extends the pseudo-Boolean function representing $v$ :

$$
\mathcal{C}_{v}\left(e_{S}\right)=v(S), \quad S \subseteq N
$$

Moreover, we clearly see that $\mathcal{C}_{v}$ is an increasing function if and only if $v$ is also an increasing function.

\section{References}

[1] C. Berge, Principes de combinatoire (Dunod, Paris, 1968). English translation: Principles of combinatorics (Academic Press, New York, 1971).

[2] A. Chateauneuf and J.Y. Jaffray, Some characterizations of lower probabilities and other monotone capacities through the use of Möbius inversion, Mathematical Social Sciences 17 (1989) 263-283.

[3] G. Choquet, Theory of capacities, Annales de l'Institut Fourier 5 (1953) 131-295.

[4] L.M. de Campos, M.T. Lamata and S. Moral, A unified approach to define fuzzy integrals, Fuzzy Sets and Systems 39 (1991) 75-90.

[5] S. Fujishige, Characterization of subdifferentials of submodular functions and its relation to Lovász extension of submodular functions. Report No. 82241-OR, Inst. für Ökonometrie und Operations Research, Bonn, 1982.

[6] M. Grabisch, On equivalence classes of fuzzy connectives : the case of fuzzy integrals, IEEE Trans. Fuzzy Systems 3 (1) (1995) 96-109.

[7] M. Grabisch, The application of fuzzy integrals in multicriteria decision making, European Journal of Operational Research 89 (1996) 445-456.

[8] M. Grabisch, $k$-order additive discrete fuzzy measures and their representation, Fuzzy Sets and Systems 92 (1997) 167-189.

[9] M. Grabisch, Alternative representations of OWA operators, in: R.R. Yager and J. Kacprzyk (eds.), The Ordered Weighted Averaging Operators: Theory and Applications, (Kluwer Academic Publisher, 1997) 73-85.

[10] M. Grabisch, J.-L. Marichal, and M. Roubens, Equivalent representations of set functions, Mathematics of Operations Research 25 (2000) 157-178.

[11] M. Grabisch, T. Murofushi, and M. Sugeno (eds.), Fuzzy Measures and Integrals - Theory and Applications. Series: Studies in Fuzziness and Soft Computing (Physica Verlag, Heidelberg, 2000).

[12] P.L. Hammer and S. Rudeanu, Boolean methods in operations research and related areas (Springer, Berlin, 1968). 
[13] U. Höhle, Integration with respect to fuzzy measures, Proc. IFAC Symposium on Theory and Applications of Digital Control, New Delhi, January 1982, 35-37.

[14] L. Lovász, Submodular function and convexity, in: A. Bachem, M. Grötschel, B. Korte (eds.), Mathematical programming. The state of the art. Bonn 1982, (Springer-Verlag, Berlin-Heidelberg-New York-Tokyo, 1983) 235-257.

[15] J.-L. Marichal, Aggregation operators for multicriteria decision aid, Ph.D. thesis, Institute of Mathematics, University of Liège, Liège, Belgium, 1998.

[16] J.-L. Marichal, Behavioral analysis of aggregation in multicriteria decision aid, in: J. Fodor, B. De Baets, and P. Perny (eds.), Preferences and Decisions under Incomplete Knowledge. Series: Studies in Fuzziness and Soft Computing Vol. 51 (Physica Verlag, Heidelberg, 2000) $153-178$.

[17] J.-L. Marichal, The influence of variables on pseudo-Boolean functions with applications to game theory and multicriteria decision making, Discrete Applied Mathematics, to appear.

[18] J.-L. Marichal, An axiomatic approach of the discrete Choquet integral as a tool to aggregate interacting criteria, IEEE Transactions on Fuzzy Systems, to appear.

[19] T. Murofushi, A technique for reading fuzzy measures (I): the Shapley value with respect to a fuzzy measure, 2nd Fuzzy Workshop, Nagaoka, Japan, October 1992, 39-48. In Japanese.

[20] T. Murofushi and S. Soneda, Techniques for reading fuzzy measures (III): interaction index, 9th Fuzzy System Symposium, Sapporo, Japan, May 1993, 693-696. In Japanese.

[21] T. Murofushi and M. Sugeno, An interpretation of fuzzy measure and the Choquet integral as an integral with respect to a fuzzy measure, Fuzzy Sets and Systems 29 (1989) 201-227.

[22] T. Murofushi and M. Sugeno, A theory of fuzzy measures. Representation, the Choquet integral and null sets, Journal of Mathematical Analysis and Applications 159 (2) (1991) $532-549$.

[23] T. Murofushi and M. Sugeno, Some quantities represented by the Choquet integral, Fuzzy Sets $\mathcal{G}$ Systems 56 (1993) 229-235.

[24] G. Owen, Multilinear extensions of games, Management Sciences 18 (1972) 64-79.

[25] G.C. Rota, On the foundations of combinatorial theory I. Theory of Möbius functions, Zeitschrift für Wahrscheinlichkeitstheorie und Verwandte Gebiete 2 (1964) 340-368.

[26] D. Schmeidler, Integral representation without additivity, Proc. Amer. Math. Soc. 97 (1986) 255-261.

[27] L.S. Shapley, A value for $n$-person games, in: H.W. Kuhn and A.W. Tucker (eds.), Contributions to the Theory of Games, Vol. II, Annals of Mathematics Studies, 28, (Princeton University Press, Princeton, NJ, 1953) 307-317. 
[28] I. Singer, Extensions of functions of 0-1 variables and applications to combinatorial optimization, Numerical Functional Analysis and Optimization 7 (1) (1984-85) 23-62.

[29] M. Sugeno, Theory of fuzzy integrals and its applications, Ph.D. Thesis, Tokyo Institute of Technology, Tokyo, 1974.

[30] R.R. Yager, On ordered weighted averaging aggregation operators in multicriteria decision making, IEEE Trans. on Systems, Man and Cybernetics 18 (1988) 183-190. 